Gut, 1975, 16, 36-41

\title{
Rectal potential difference in the diagnosis of aldosterone excess
}

\author{
D. G. BEEVERS, J. J. MORTON, M. TREE, AND JESSIE YOUNG \\ From the Medical Research Council Blood Pressure Unit, Western Infirmary, Glasgow
}

SUMMARY Rectal potential difference (pd) is directly related to the plasma aldosterone concentration, and rises when aldosterone is stimulated by sodium deprivation. However, when the measurement of rectal pd was tested at a screening test for hyperaldosteronism in 19 hypertensive subjects, four of the eight with primary hyperaldosteronism had a normal pd and four of the eight without aldosterone excess had an abnormally raised potential difference. The technique cannot therefore be recommended as a routine screening test for hyperaldosteronism. No relationship was found between rectal pd and hypertension associated with excess of deoxycorticosterone. Rectal pd rises in response to the mineralocorticoid-like agent carbenoxolone.

Investigations necessary to sustain a diagnosis of an aldosterone-secreting adrenocortical adenoma are complex, and usually necessitate referral of the patient to a specialized centre. Although the disease is probably not as common as previously postulated (Conn, 1955; Conn, Cohen, Rovner, and Nesbit, 1965; Ledingham, Bull, and Laragh, 1967), a simple screening test would be of value.

Aldosterone exerts its sodium-retaining action on organs other than the kidney and these include the sweat glands (McConahay, Robinson, and Newton, 1964), salivary glands (Wotman, Goodwin, Mandel, and Laragh, 1970), and the colon (Levitan and Ingelfinger, 1965; Holt, Finck, Zintel, and Crouse, 1965; Richards, 1969). Investigations of these 'target organs' have generally failed to produce a reliable test of aldosterone excess. Samples of sweat or saliva are difficult to collect, and the faecal electrolyte concentrations may contain a proportion derived from undigested food fibres and small intestinal enterocytes (Wrong, Gibson, Morrison, $\mathrm{Ng}$, and Howard, 1965), although the technique of faecal dialysis in vivo (Charron, Leme, Wilson, Ing, and Wrong, 1969), may overcome some of these problems.

Recently a simple potentiometer and electrode have been devised (Edmonds and Cronquist, 1970; Edmonds and Godfrey, 1970; Edmonds and Richards, 1970; Archampong and Edmonds, 1972), capable of measuring the potential difference (pd) generated by the transport of sodium ions across the

Received for publication 18 September 1974. rectal mucosa. Initial results using this technique (Edmonds and Richards, 1970; Richards, 1971; Lancet, 1973) in the diagnosis of primary hyperaldosteronism have been encouraging. The purpose of the present study was to assess the method in a series of hypertensive subjects, some with and some without primary hyperaldosteronism.

\section{Patients and Methods}

All 19 hypertensive subjects were inpatients receiving a constant diet for five days with the sodium and potassium intakes fixed at a point within the ranges of 120 to 140 m-equiv and 40 to $60 \mathrm{~m}$-equiv respectively. With the exception of the two patients with secondary hyperaldosteronism, none were receiving antihypertensive drugs and none had taken diuretics, potassium supplements, liquorice, carbenoxolone, or oral contraceptives within the previous four weeks. All had had a diastolic blood pressure of $100 \mathrm{~mm}$ $\mathrm{Hg}$ or more while attending the outpatient department.

The subjects with 'essential hypertension' were those whose mean plasma renin concentration was 6 units/litre or more. 'Low renin hypertension' was taken as a mean plasma renin concentration below an arbitrary level of 6 units/litre. Plasma aldosterone concentration was normal in both of these groups. All had normal intravenous urograms, femoral pulses, and urine metadrenaline, and none had clinical or biochemical evidence of Cushing's syndrome. The table shows the mean value for blood pressure, serum electrolytes, plasma renin, plasma 


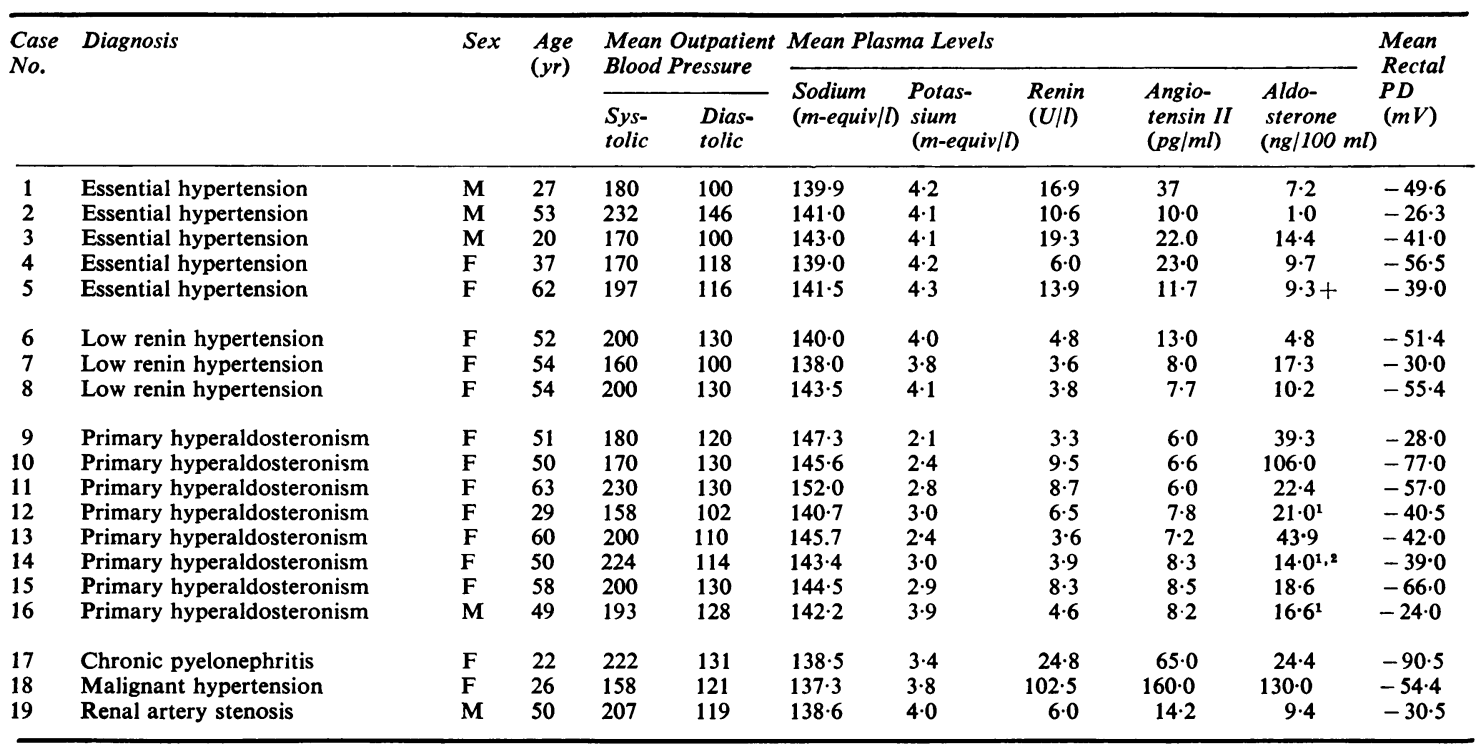

Table Clinical features of hypertensive subjects studied

${ }^{1}$ Intermittent high levels.

${ }^{2}$ Elevated plasma deoxycorticosterone.

angiotensin II, and plasma aldosterone concentrations together with the rectal potential difference.

Cases 9 to 16 all had primary hyperaldosteronism as defined previously (Brown, Davies, Ferriss, Fraser, Haywood, Lever, and Robertson (1972a) and six (cases 9 to 14) have subsequently undergone resection of an adrenocortical adenoma. One patient (case 15) has not undergone surgery but analysis of the data by quadric analysis gave odds of 2.4 to 1 on the diagnosis of adrenocortical adenoma (Ferriss, Brown, Fraser, Kay, O'Muircheartaigh, Robertson, Symington, and Lever, 1970). Case 16, a subject with hypertension with intermittently high plasma levels of aldosterone, also had elevated plasma levels of deoxycorticosterone (DOC). At operation no adenoma was found, but histology showed adrenocortical micronodular hyperplasia.

Cases 17 and 18 had secondary hyperaldosteronism, one with essential hypertension in the malignant phase and one with hypertension associated with chronic pyelonephritis. Both were receiving adrenergic blocking agents at the time of examination.

Case 19 was a subject with hypertension with complete occlusion of the left renal artery, in whom plasma levels of renin, angiotensin II, and aldosterone were normal.

The eight control subjects were patients in the medical wards with diastolic blood pressure consistently below $95 \mathrm{~mm} \mathrm{Hg}$ and were undergoing investigation of other diseases, including peptic ulceration and obesity. None had evidence of renal or adrenal disease and none were receiving diuretics or carbenoxolone therapy (Beevers, 1973).

Blood for measurement of plasma renin (Brown, Davies, Lever, Robertson, and Tree, 1964) (normal range: 4-18 u/l), plasma angiotensin II (Düsterdieck and McElwee, 1971) (normal range 5-35 pg/ml), and plasma aldosterone (Fraser and James, 1968; Fraser, Guest, and Young, 1973) (normal range 0-18 ng/100 $\mathrm{ml})$ concentrations were taken after overnight recumbency and fasting, on three occasions, and the mean values calculated.

The potentiometer and reference and probe electrodes were as described by Archampong and Edmonds (1972). The reference electrode was applied to the skin over a bleb raised by intradermal injection of isotonic saline. This technique abolishes the pd across the skin under the electrodes. The technique has been shown to produce a normal range which is similar to that obtained using intravenous saline lead as the indifferent electrode (Dalmark, 1970). The probe electrode, wetted with isotonic saline, was applied to the rectal mucosa under direct vision through an $8 \mathrm{~cm}$ proctoscope and a voltmeter reading of the potential difference between the rectal mucosa and the reference electrode was made.

A series of 10 to 20 readings was taken on each occasion at different points around the circumference 
of the rectum. From this figure was subtracted the resting pd across the electrodes when both were placed in an isotonic saline bath. The resultant figure was taken as the actual rectal potential difference. The mean rectal potential difference for eight normotensive control subjects was $-34.8 \mathrm{mV}$ (range -28 to $-42 \mathrm{mV}$ ). The coefficient of variation for 10 to 20 repeated measurements in each of eight subjects varied from $7 \cdot 7$ to $23 \%$ (mean $15.9 \%$ ). Replicate measurements on the same subject on separate occasions showed a coefficient of variation of $9.5 \%$.

\section{Results}

RESPONSE TO DIETARY SODIUM DEPLETION

The response of a subject with essential hypertension to dietary sodium depletion was studied. The sodium intake was reduced to $11 \mathrm{~m}$-equiv daily and there was a rapid reduction of the urinary sodium excretion. This was associated with a rise in plasma levels of renin, angiotensin II, and aldosterone, and the rectal pd, initially normal, rose to very high levels (fig 1).

\section{RECTAL PD AND CONCURRENT PLASMA} ALDOSTERONE CONCENTRATION

Including all normotensive and hypertensive subjects where there were concurrent measurements of plasma aldosterone concentration and rectal pd, a correlation was found between pd and plasma aldosterone concentration. This was a significant but not particularly close relationship. As fig 2 shows, patients with elevated rectal pd may have normal plasma aldosterone concentration.

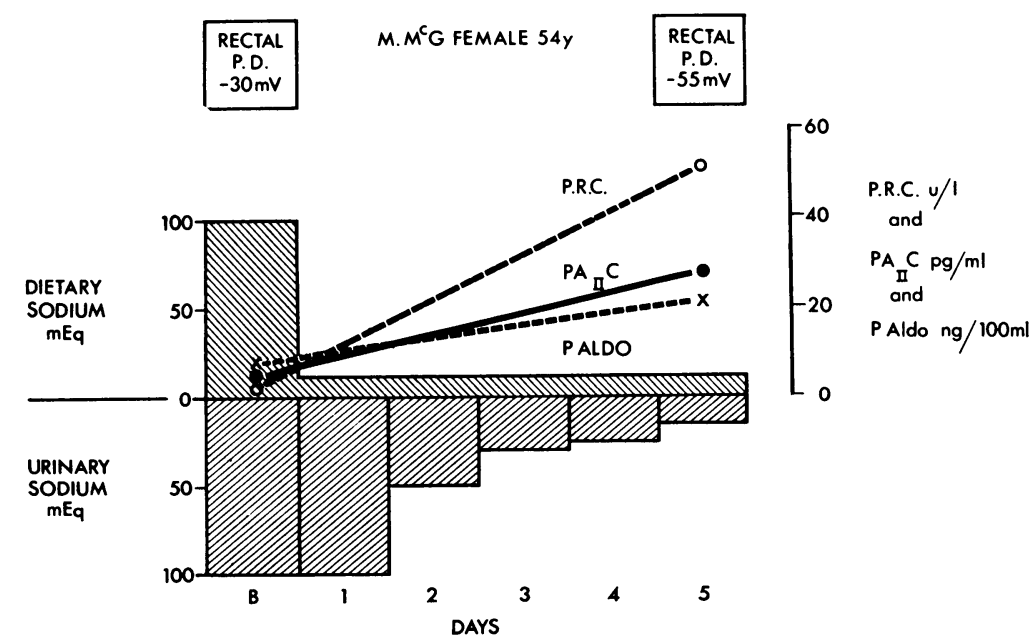

Fig 1 The response of rectal pd, plasma concentrations of renin, angiotensin $I I$, and aldosterone to sodium

depletion.

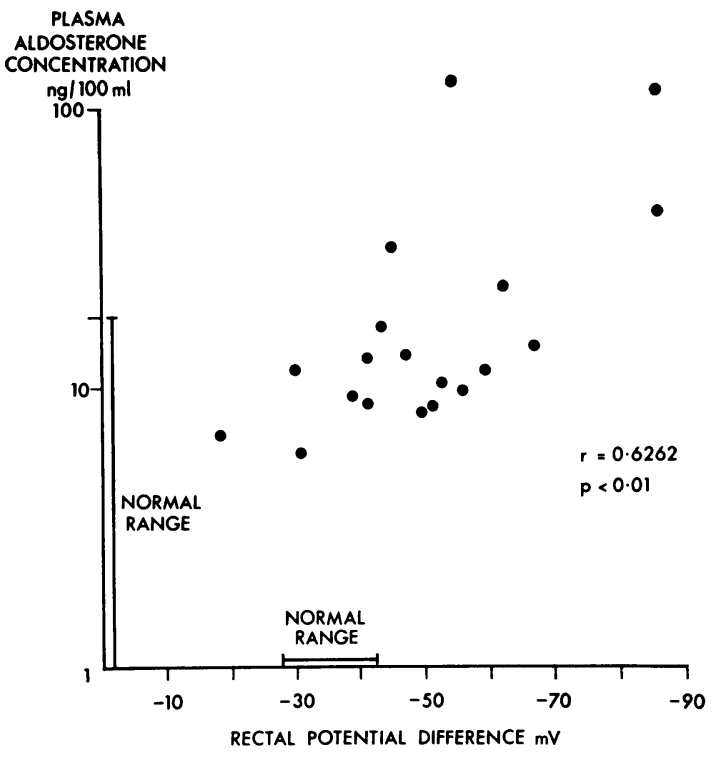

Fig 2 Relation between concurrent measurements of rectal pd and plasma aldosterone concentration.

RECTAL PD IN THE DIAGNOSIS OF HYPERALDOSTERONISM

In patients with essential hypertension the mean rectal pd was $-42.5 \mathrm{mV}( \pm 11.4 \mathrm{SD})$. As can be seen from the table and fig 3 , some of these subjects had potential differences clearly greater than normal. In patients with low-renin hypertension in whom there was no evidence of aldosterone excess, elevated levels of rectal pd were also obtained. The mean rectal pd for this group was $-45.6 \mathrm{mV}( \pm 13.7 \mathrm{SD})$ 


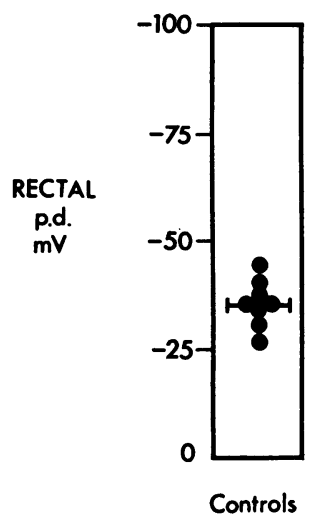

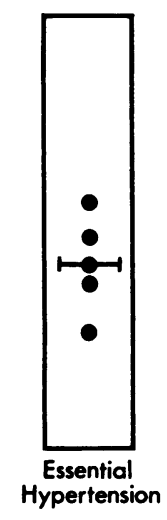

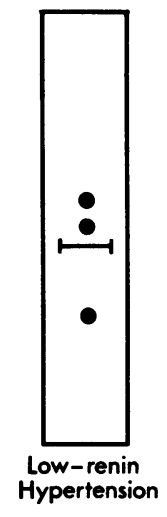

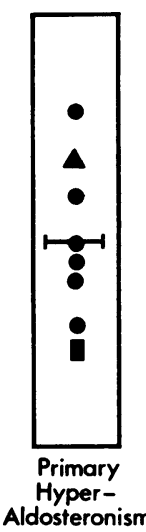

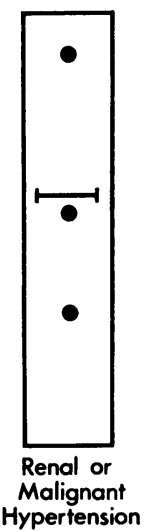

Fig 3 Rectal potential difference in different forms of hypertension. All patients with primary hyperaldosteronism underwent resection of an adrenal adenoma except $\square$, who had adrenal hyperplasia, and $\boldsymbol{\Delta}$, who has not yet undergone surgery.
Of the eight subjects with primary hyperaldosteronism, the mean rectal pd was $-47.3 \mathrm{mV}$ $( \pm 18.3 \mathrm{SD})$. As can be seen from the table, four of these subjects had levels of rectal pd within the normal range. Both cases with secondary hyperaldosteronism had greatly elevated levels of rectal pd, but no such elevation was seen in the subject with renal artery stenosis without hyperaldosteronism.

\section{RESPONSE TO FLUDROCORTISONE}

In seven hypertensive subjects (one with essential hypertension, two with low-renin hypertension, and four with primary hyperaldosteronism) the rectal pd was measured before and while receiving fludrocortisone, $0.4 \mathrm{mg}$ bd for three days. Failure of the rectal pd to rise was seen in two subjects with lowrenin hypertension and two with primary hyperaldosteronism. A marked rise in pd was seen in one subject with essential hypertension and in two patients with primary hyperaldosteronism.

\section{PATIENTS WITH EXCESS}

\section{DEOXYCORTICOSTERONE}

Elevated plasma levels of deoxycorticosterone were found in three hypertensive subjects (one with essential hypertension, one with an adrenocortical adenoma and one with adrenocortical micronodular hyperplasia). In none was the rectal pd elevated, and no relation was found between rectal pd and plasma concentration of deoxycorticosterone.

\section{RESPONSE TO CARBENOXOLONE}

The action of the mineralocorticoid-like agent carbenoxolone sodium was tested on one normotensive subject with a gastric ulcer. While on this occasion he developed neither hypertension, hypokalaemia, nor oedema, his rectal pd rose from the normal range to very high levels (fig 4) and there

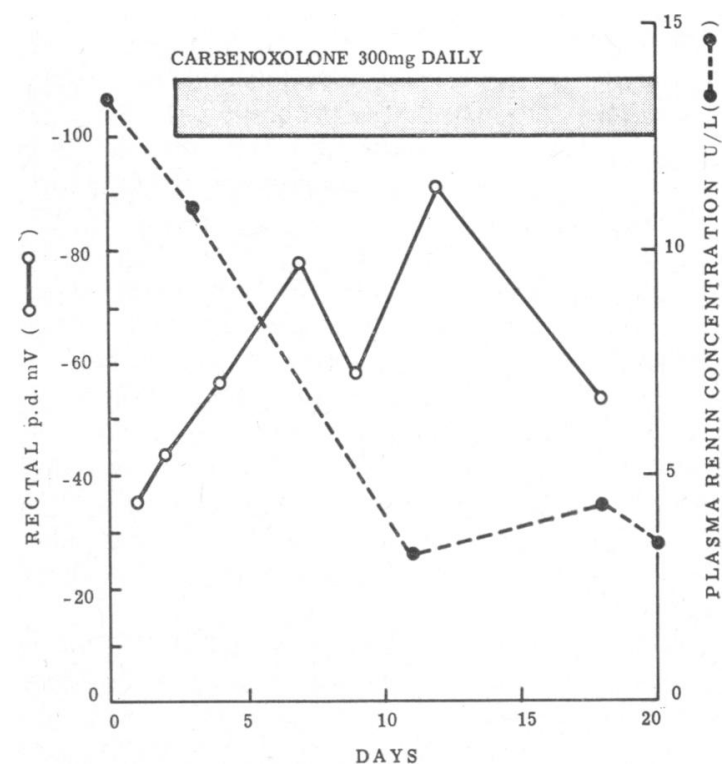

Fig 4 Response of rectal pd and plasma renin concentration to oral carbenoxolone therapy (reproduced by permission of the Editor, Lancet).

was a concurrent fall of plasma renin concentration to subnormal levels. The plasma aldosterone concentration, initially $6.5 \mathrm{ng} / 100 \mathrm{ml}$, fell to $4.3 \mathrm{ng} / 100$ $\mathrm{ml}$.

\section{Discussion}

The technique described here for measuring rectal pd was similar to that used by other workers (Archampong and Edmonds, 1972) and the mean for normal subjects was also similar.

The response of the rectal pd to sodium depletion 
confirms that the colonic mucosa is responsive to changes in aldosterone concentration. While a significant relationship was found in the present series between concurrent measurements of rectal pd and plasma aldosterone concentration, the measurement of rectal pd was not an infallible screening test for primary hyperaldosteronism, as false negative results were obtained. Furthermore, high levels of rectal pd were obtained from patients without aldosterone excess. While in some cases of low-renin hypertension the presence of an as yet unidentified mineralocorticoid may be invoked (Beevers, Brown, Ferriss, Fraser, Lever, and Robertson, 1973) which might cause elevation of the rectal pd, this does not readily explain the high levels seen in some patients with essential hypertension and normal renin.

The response of the rectal pd to carbenoxolone therapy, together with the suppression of renin and aldosterone, confirms the mineralocorticoid-like action of this drug. The hypertension and oedema sometimes demonstrated is probably due to sodium retention, and it is significant that this action is blocked by the steroidal antagonist spironolactone, although this also inhibits the ulcer-healing effect.

The finding of elevated plasma levels of deoxycorticosterone here in a subject with essential hypertension, and in cases of low renin hypertension (Brown, Ferriss, Fraser, Lever, Love, Robertson, and Wilson, 1972b), as well as in hyperaldosteronism (Biglieri, Slaton, Schambelan, and Kronfield, 1968), remains to be fully explained. However, this high level of mineralocorticoid cannot account for the false positive result in essential hypertension when the rectal pd is used as a screening test for excess aldosterone.

The failure of treatment with fludrocortisone to depress urinary aldosterone excretion rates has been suggested as indicating an adrenocortical adenoma rather than hyperplasia (Biglieri, Stockigt, and Schambelan, 1970). If this is so, failure of the rectal pd to alter after fludrocortisone might be a diagnostic test for adenoma. However, fludrocortisone raises the rectal pd in its own right in normal subjects (Edmonds and Richards, 1970) and the elevation of the rectal pd seen in some, but not all, of the subjects with adrenocortical adenoma during fludrocortisone therapy may be attributable to this.

Four of our patients with primary hyperaldosteronism, who subsequently underwent excision of an adrenocortical adenoma, had intermittently normal plasma aldosterone concentrations, and this may well explain the normal values for rectal pd obtained in some cases. Intermittency of hyperaldosteronism is not unusual (Biglieri, Slaton, Kronfield, and Deck, 1967) and frequent estimations may be necessary before hyperaldosteronism is detected.
Recently a patient has been described (Richards, Jones, and Peart, 1973) with hypertension and hypokalaemia, due to an adrenocortical adenoma in whom the plasma renin activity was low, but plasma aldosterone concentration was of borderline levels on three occasions. In this patient, the rectal pd was normal. It was suggested, therefore, that profound intracellular potassium depletion may prevent the colonic mucosa from manifesting a raised potential difference. This would certainly explain the normal values in some of our cases of primary hyperaldosteronism, as hypokalaemia is a feature of this syndrome. Possibly, if the patients were potassium repleted, then the rectal potential difference might rise to abnormal levels, but the test would then cease to be an instant screening test, and interpretation of a normal value may be misleading in the diagnosis of excess aldosterone.

We are grateful to Dr C. J. Edmonds for advice and the measurement of rectal pd in case 9, and G. D. Searle \& Co Ltd, High Wycombe, Bucks, for financial support and equipment.

\section{References}

Archampong, E. Q., and Edmonds, C. J. (1972). Effect of luminal ions on the transepithelial electrical potential difference of human rectum. Gut, 13, 559-565.

Beevers, D. G., Brown, J. J., Ferriss, J. B., Fraser, R., Lever, A. F., and Robertson, J. I. S. (1973). The use of spironolactone in the diagnosis and the treatment of hypertension associated with mineralocorticoid excess. Amer. Heart J., 86, 404-414.

Beevers, D. G. (1973). Mineralocorticoids and rectal potential difference. Lancet, 2, 682-683.

Biglieri, E. G., Slaton, P. E., Jr., Kronfield, S. J., and Deck, J. B. (1967). Primary aldosteronism with unusual secretory pattern. J. clin. Endocr., 27, 715-721.

Biglieri, E. G., Slaton, P. E., Schambelan, M., and Kronfield, S. J. (1968). Hypermineralocorticoidism. Amer. J. Med., 45, 170-175.

Biglieri, E. G., Stockigt, J. R., and Schambelan, M. (1970). A preliminary evaluation for primary aldosteronism. Arch. intern. Med., 126, 1004-1007.

Brown, J. J., Davies, D. L., Lever, A. F., Robertson, J. I. S., and Tree, M. (1964). The estimation of renin in human plasma. Biochem. J., 93, 594-600.

Brown, J. J., Davies, D. L. Ferriss, J. B., Fraser, R., Haywood, E., Lever, A. F., and Robertson, J. I. S. (1972a). Comparison of surgery and prolonged spironolactone therapy in patients with hypertension, aldosterone excess and low plasma renin. Brit. med. J., 2, 729-734.

Brown J. J., Ferriss, J. B., Fraser, R., Lever, A. F., Love, D. R., Robertson, J. I. S., and Wilson, A. (1972b). Apparently isolated excess deoxycorticosterone in hypertension: a variant of the mineralocorticoid excess syndrome, Lancet, 2, 243-247.

Charron, R. C., Leme, C. E., Wilson, D. R., Ing, T. S., and Wrong, O. M. (1969). The effect of adrenal steroids on stool composition, as revealed by in vivo dialysis of faeces. Clin. Sci., 37, 151-167.

Conn, J. W. (1955). Primary aldosteronism, a new clinical syndrome. J. Lab. clin. Med., 45, 3-17.

Conn, J. W., Cohen, E. L., Rovner, D. R., and Nesbit, R. M. (1965). Normokalemic primary aldosteronism. J. Amer. med. Ass., 193, 200-206.

Dalmark, M. (1970). The transmucosal electrical potential difference of rectum in the unanaesthetised man. Scand. J. Gastroent., 2, 277-282.

Düsterdieck, G., and McElwee, G. (1971). Estimation of angiotensin II concentration in human plasma by radioimmunoassay: 
some applications to physiological and clinical states. Europ. J. clin. Invest., 2, 32-38.

Edmonds, C. J., and Cronquist, A. (1970). A simple millivoltmeter and electrodes for measurement of rectal electrical potential in man. Med. Biol. Engng., 8, 409-410.

Edmonds, C. J., and Godfrey, R. C. (1970). Measurement of electrical potentials of the human rectum and pelvic colon in norma and aldosterone treated patients. Gut, 11, 330-337.

Edmonds, C. J., and Richards, P. (1970). Measurement of rectal electrical potential difference as an instant screening test for hyperaldosteronism. Lancet, 2, 624-627.

Ferriss, J. B., Brown, J. J., Fraser, R., Kay, A. W., Neville, A. M. O'Muircheartaigh, I. G., Robertson, J. I. S., Symington, T. and Lever, A. F. (1970). Hypertension with aldosterone excess and low plasma-renin: preoperative distinction between patients with and without adrenocortical tumour. Lancet, 2, 995-1000.

Fraser, R., Guest, S., and Young, J. (1973). A comparison of doubleisotope derivative and radioimmunological estimation of plasma aldosterone concentration in man. Clin. Sci., 45, 411-415.

Fraser, R., and James, V. H. T. (1968). Double isotope assay of aldosterone, corticosterone and cortisol in human peripheral plasma. J. Endocr., 40, 59-72.

Holt, P. R., Fink, D. L., Zintel, H. A., and Crouse, J. (1965). Effects of aldosterone on colonic transport of sodium in dogs. Clin. Res., 13, 31 .
Lancet (1973). Potential difference of gut epithelia. (Editorial.) Lancet, 2, 424-425.

Ledingham, J. G. G., Bull, M. B., and Laragh, J. H. (1967). The meaning of aldosteronism in hypertensive disease. Circulat. Res., 21, Suppl. 2, 177-187.

Levitan, R., and Ingelfinger, F. J. (1965). Effect of d-aldosterone on salt and water absorption from the intact human colon. J. clin. Invest., 44, 801-818.

McConahay, T. P., Robinson, S., and Newton, J. L. (1964). d-Aldosterone and sweat electrolytes. J. appl. Physiol., 19, 575-579.

Richards, P. (1969). Clinical investigation of the effects of adrenal corticosteroid excess on the colon. Lancet, 1, 437-442.

Richards, P. (1971). Detection of mineralocorticoid excess using spironolactone. In The Medical Uses of Spironolactone, edited by G. M. Wilson, pp. 17-26. Excerpta Medica, Amsterdam.

Richards, P. (1973). Mineralocorticoids and rectal potential difference. Lancet, 2, 798-799.

Richards, P., Jones, M. B. S., and Peart, W. S. (1973). Periodic hypokalaemic paralysis, adrenal adenoma, and normal colonic transport of sodium and potassium. Gut, 14, 478-484.

Wotman, S., Goodwin, F. J., Mandel, I. D., and Laragh, J. H. (1969). Changes in salivary electrolytes following treatment of primary aldosteronism. Arch. intern. Med., 124, 477-480.

Wrong, O. M., Metcalfe-Gibson, A., Morrison, R. B. I., Ng, S. T., and Howard, A. V. (1965). In vivo dialysis of faeces as a method of stool analysis. 1 . Technique and results in normal subjects. Clin. Sci., 28, 357-375. 